\title{
An Overview of Autonomous Loading of Bulk Material
}

\author{
Ahmad Hemami and Ferri Hassani \\ PhD, Department of Mining and Materials Engineering, McGill University, Montreal, Canada, H3A 2A7, \\ \{ahmad.hemai, ferri.hassani\}@mcgill.ca
}

\begin{abstract}
Autonomous loading implies a fully automated scenario in which automated excavating machines, such as front-loaders, load themselves from a heap of bulk material and deliver to the dumping site. The process comprises all the functions of loading, navigating, obstacle detection and avoidance and unloading to be automated and controlled by a supervisory computer. Autonomous loading benefits a number of industries such as construction and mining, from economical view point as well as other concerns like operators safety when the workplace is not hazard free. Despite all the benefits and despite considerable amount of research on the subject, there are no commercially available systems that can be purchased and put to work. In addition to a breakdown of all the tasks that need to be automated and the difficulties involved, this paper reviews and reports the various research and/or development activities that have been carried out during the past two decades on the subject.
\end{abstract}

Keywords: loading, automated, autonomous, excavation, autoloading

\section{Introduction}

Over the past three decades a rather significant amount of work has been carried out regarding automation of excavating machinery. It would be fascinating in an earth moving operation to benefit from a similar scenario as available in manufacturing, where robot manipulators are programmed to perform many of the desired tasks. Such a scenario is, however, much more complex than that of manufacturing. Such capability is called autonomous loading, or it can be referred to as autonomous excavation or other similar terms. The complexity stems from the nature of the operation and the fact that there are various excavating machines and that each one must be dealt independently. Moreover, there are a number of tasks involved in autonomous loading and, in order to have a successful operation all of these tasks must be automated.

Figure 1 illustrates a very simple loading site in which there is only one excavator that must transfer the earth from a heap to a single unloading point. The problem would have been much easier if rotary excavators and conveyer belts were employed. But, the problem under investigation is as it is shown in Figure 1, where cyclic excavators such as backhoe or front-end loader have to interact with a medium that is heterogeneous and whose behavior is stochastic.

In the loading scenario shown the following facts must be taken into account and the corresponding problems must be resolved:

1- The heap to load from has a dynamic structure that changes with each cycle of operation. The loading point, thus, is not fixed and needs to be determined for each cycle of loading.

2- The loading must be successful each time no matter what forces the loading tool experiences from the medium. In this case bucket filling is the ultimate objective. In other cases a different objective may be involved, instead. For example when a ditch is dug by a backhoe, in the last run it is necessary to have a flat surface. The safe operation and safety of the machine must always be respected. In addition, the efficiency of the work cannot be overlooked.

3- When the bucket is filled, the excavator must navigate to the unloading point. This implies deciding on the trajectory to follow, while detecting and avoiding any obstacle. The speed of this transport must be reasonable, meaning that it should take about the same (ideally less) time that it takes for an operator. 
4- Unloading to the dumping point is to be with no collision with any carrying truck, belt or so on. The whole load must be delivered (In the case of wet soil, for instance, it is possible that a good portion of the material is carried back without being emptied.)

5- Operation (3) must be repeated while a new trajectory is planned and followed, as required.

6- In all the above operations the environment conditions, such as the slope of the terrain and the temperature (and the gravity constant, if not on Earth), play a role in the operation, which cannot be ignored and must be considered.

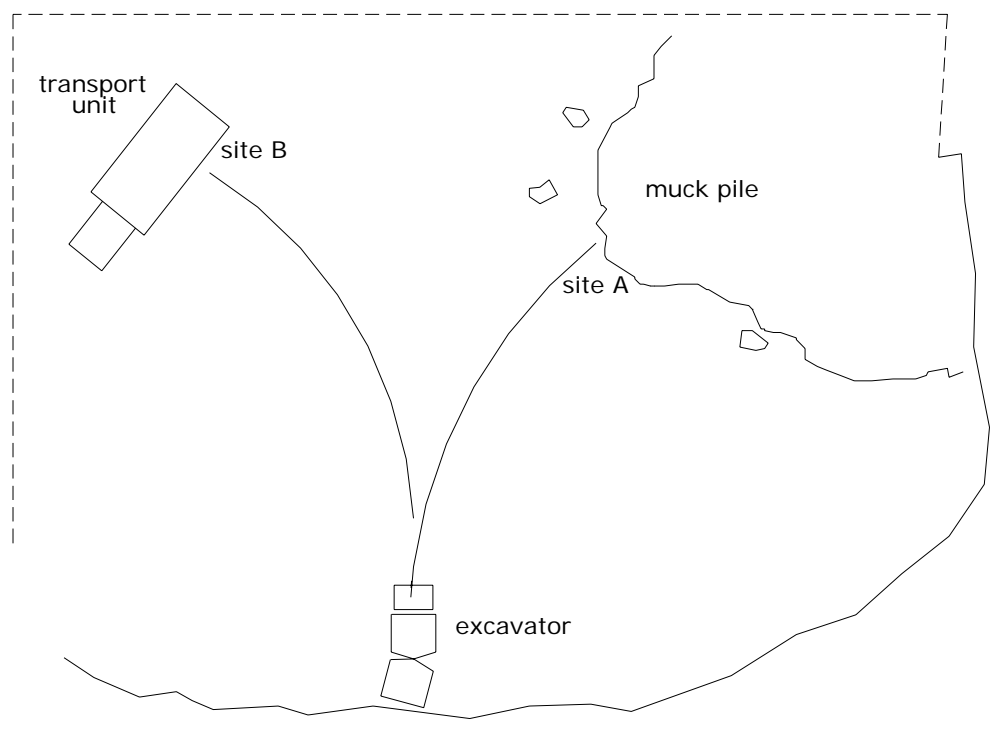

Fig.1- A simple scenario of autonomous loading

A fully autonomous system implies that all the operations are automated and controlled by a supervisory computer. Semi-automated systems, however, are possible and have been practiced for simple cases. For example, if the loading is done by an operator but the navigation between a specific area near the loading site to a specific area near the unloading site is automated. This is useful when there is a long enough distance between the two sites.

\section{Main Tasks}

The above example reveals the main tasks involved in autonomous loading. Basically these tasks are:

(a) Excavation (or loading)

(b) Navigation

(c) Obstacle detection

(d) Obstacle avoidance

The main issue in this paper is the first task which, despite numerous works on the subject, has not yet yielded a successful result. Navigation has received tremendous attention, not only as a task for autonomous loading but as part of mobile robots in manufacturing. Mobile robots, contrary to Automated Guided Vehicles (AGV) which have been employed in manufacturing much earlier, have the advantage that they do not required buried wires as well as the fact that they are programmable and, thus, flexible in this sense. Also, the work regarding obstacle detection and avoidance is widely treated in mobile robot technology.

The extension of the mobile robot technology to non-manufacturing application started with the automated navigation of a Load-Haul-Dump unit in an underground mine between a stope (where the fragmented rock is loaded for transportation) and the ore-pass (where the dump-track unloads its load). A roof mounted reflected line and a camera have been used to guide the vehicle along the mine passages (A schematic of a Load-Haul-Dump unit, which from a loading function viewpoint resembles a front-end loader, is shown in the next section). An LHD (Load-Haul-Dump) is a wheeled vehicle which is made merely for underground mines. This work was carried out by Noranda Technology Centre in Canada. This 
unit was not equipped with obstacle detection and avoidance capability to protect it from the boulders that could have fallen on the route. Later on the reflecting tape was replaced by a laser system.

\section{Excavating machines}

Figure 2 shows the most popular excavating machines used in construction sites, road work and mining. Automation of excavation or auto-loading implies that the operation of an excavator is automated and the machine can load its bucket with the proper motion and interaction with the medium. It is obvious that such a capability requires a good deal of artificial intelligence to be embedded into a machine in the form of various sensors, algorithms to interpret the sensory information and control laws based on which the machine moves itself or its bucket. As can be seen, each machine has its own characteristic dictated by its structure, meaning that it must be treated differently from the rest for the purpose of turning to an automated device.

What is common between all the various types of excavating machines is the fact that all must interact with the medium to be loaded or excavated. They have to overcome the resistive force experienced by their bucket when penetrating into a medium. In most of the cases the penetration into the medium is in the form of cutting (and breaking), digging and scooping.
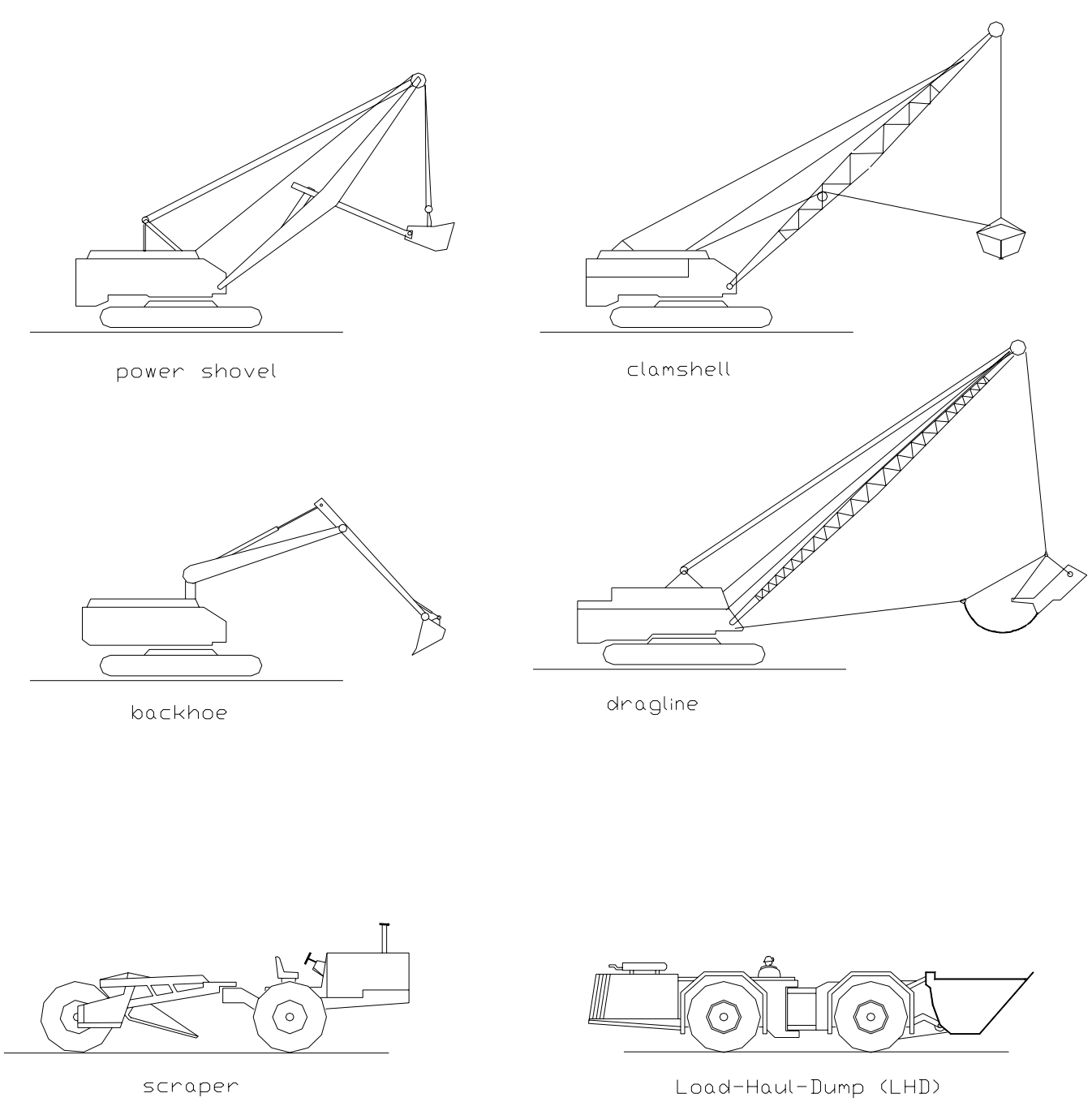

Fig. 2- Various excavating machines

Any automated system works based on the measurement of the main variable(s), forming the error based on the comparison between actual and desired value(s) and a control policy based on which a feedback signal is generated for the control input. The principal question in auto-loading is "What must be measured and 
what must be controlled". For all excavators, since motion (of the bucket) is involved, a robotic approach (That is, modeling the excavator as a robot manipulator) is an appropriate one. In this sense, the effect of each actuator on the bucket can be defined. If a trajectory for the motion of the bucket is defined, then the variation of each actuator and its speed can be calculated. Furthermore, the relationship between the forces acting on the bucket and those at the actuator level may be defined.

Although it may sound perfect, dealing with an excavator is much more complicated compared to a robot manipulator, because of the following reasons:

1- The forces from the medium to the bucket can have enormous variation not only from one cycle of loading to another, but within the same cycle of operation. These forces can easily become very large at the actuator level and, as a result, any of the actuators may reach its maximum capacity. This is more likely to happen with non-heterogeneous material like a mixture of different soils with different size boulders in it (Fragmented rock, for instance).

2- The cutting tool (bucket) always finds the least resistive path to follow.

3- The structure of the excavator is much more flexible compared to a robot manipulator, specially for the large size payload at the bucket. The body of the machine thus can deform under the influence of large resistive loads mentioned above.

4- As a result of (1) to (3), the trajectory followed by the bucket can significantly deviate from the desired.

In manual operation of any of the excavators shown in Figure 1, and other machines with similar structure, an operator uses his judgment and skills to load the bucket. The motion of the bucket could consist of back and forth maneuvers and repeats. In other words, bucket motion is composed of a series of logically induced smaller motions, and can be very complex. Indeed, an operator judges the forces of medium interaction on the bucket, based on what he sees and hears the load inside or in front of the bucket and the vibrations he feels from the machine. He then moves the bucket accordingly.

Obviously, it is quite difficult if not impossible to enhance a machine with the same type of capability and intelligence as an operator has. Thus a systematic analysis of the way and the extent to which an excavator must be enhanced with sensors and artificial intelligence has been the main focus of the on-going research on the subject of automation of excavation, thus far.

\section{Automated excavation history in brief}

The start of the work directly targeted for automation of the process of excavating by one of the nonrotary machines shown in Figure 1 goes back to late 80's and onward, as the reported work starts from early 90's. The prevailing force, one can say, was for both commercial benefits and extraterrestrial discovery aspects [Bernold (1991)]. The earliest research started in Soviet Union as exemplified by the work of Mikhirev (1986). In the west, the work associated with the commercial side are those at Lancaster University, UK [Seward et al (1988), (1992)] and Purdue University [Vähä et al (1991)] towards the automation of a backhoe for digging ditches, and the work at Noranda Technology Centre (NTC), in Montreal, Canada, for automation of both loading and transportation for a Load-Haul-Dump (LHD) unit. LHD is extensively used in underground mines. The nearest category of on-ground excavators to an LHD is a wheeled frontend loader. The work at Lancaster University was more of experimental nature on a small backhoe equipped with force sensors [Bradley \& Seward (1995)]. Some success was reported for digging trenches in soft soil. On the other hand, the work at Purdue University involved was of a more analytic nature, such as kinematic and dynamic modeling of backhoe [Cetto and Koivo (1995)]. This analysis is necessary for a robotic approach to tackle the problem. The work at NTC was more involved, since it was directly supported by industry. The approach was to make a machine perform the same sort of motion that a skilled operator did during loading. A real size LHD was equipped with as many sensors that could be accommodated to record the various parameters such as pressure and motions during a loading practice. This was an ad-hoc approach and did not lead to any success. A vibratory motion was later blended into the main motion of the bucket. This led to some success, but it could never be claimed that such a machine could perform as efficient as an operator. The results of the work (or better say, the technical details) on auto-loading were not published at all, as the trend in industry is. NTC was, nevertheless, successful in development of the automated guidance system in underground mines employing a roof mounted reflected tape [Hurteau et al (1992)]. A systematic approach was later begun [Hemami (1993), (1994a), (1994b)] at The Canadian Centre 
for Automation and Robotics in Mining (CCARM). This work continued with a lower pace later on because of luck of financial support. Another player joining the group later was Carnegie Mellon University [Singh (1991), Luengo (1998), Cannon (1999)] At Robotic Institute a good deal of research on relevant subjects begun [Rowe (1999), Stentz et al (1999a), (1999b)]. . Research activities expanded to University of Arizona [Lever \& Wang (1995), Shi et al (1996)] and Tohoku University in Japan [Takahashi et al (1995), (1999)]. More work on the subject includes the research activities in Australia [Corke et al (1997), Roberts et al (1999)].

There are other institutions that are involved in research on this topic, as can be seen from the work of Marshall (2001), Mrad et al (2002), Zweiri et al (2002) Serata et al (2003), Serata et al (2005). The subject still demands further work and funded research. Some progress is continuously reported such as the latest works [Richardson-Little \& Damaren (2008), Vahed et al (2008)] though at a slow pace. But strong industry support can speed up the progress.

\section{Relevant research work on excavation automation}

In the previous sections a brief but comprehensive introduction to subject was presented. There are a number of topics that are relevant to the subject and the research works by various groups are devoted to one or more of these. It is possible that when an automated system is finally available it will not use all of the findings of the research. The research so far has been on one of the following:

1- Modeling of the excavator as a robot manipulator, or determining the kinematic relationships between the actuator motion and the bucket motion.

2- Determining the force and velocity relationships between the bucket and the actuators.

3- Trajectory studies and planning for the bucket motion.

4- Dynamic modeling of the bucket and actuators.

5- More specific studies of the hydraulics related to bucket motion (Since almost all the excavators work with hydraulic power).

6- Computer modeling of the heap of soil and dynamics of changes due to excavation.

7- Automatic recognition of the shape of the heap and decision making for starting point for loading.

8- Automatic recognition of the environment geometry (Inside a mine, for example)

9- Analysis of the composition of the fragmented rock (size variation) and its effect on loading.

10- Analysis of the forces (various force components) of interaction of bucket and medium.

11- Analysis and formulation of the cutting force and the mechanism of material failure when subject to bucket forces.

12- Use of computer vision to estimate the amount of loaded material.

13- Methods to control the motion (Control strategy).

14- Computer simulation of the process.

The references cited in this paper are examples of the work that has been carried out during the past three decades. By no means can one claim that this review is complete and all the previous works are addressed. From those addressed and their references one can count more than 500 technical papers on the subject. A good review of the various pertinent works before 1997 is presented by Singh (1997).

Since during an excavation task there is an exchange of active and resistive forces, from the bucket and the medium respectively, significant research has been devoted to identifying, analysing and formulation of forces that are involved in the process. Many formulations are proposed, but they have not been verified and are not in accord with each other [Hemami et al (1994c)]. A good review and comparison of the various proposed theories for formulating the earthmoving forces can be found in Blouin et al (2001).

As far as the control philosophy is concerned, there are not many proposed methods to control the process yet, and none has proven to provide an acceptable solution. Indeed, there are only three main approaches. Bullock et al (1990) were the first to propose a two-level control based on the measurement of the excavation forces during the operation. Some researchers believe that a controller does not need to be based on the analysis or measurement of the forces and, therefore, controllers based on fuzzy logic instructions can work [Ha et al (2002), Ha \& Rye (2004)]. This second approach was tested earlier by some other groups [Shi et al (1996)]. A third approach is that any action be based on the maximum power of the 
excavator. In other words, an excavator is always working with maximum output power on the bucket. This is the approach implemented to LUCIE [Seward et al (1992), Bradley \& Seward (1995)].

Any proposed method must be proven by implementation to real tasks and in the realistic conditions. In other words, more experimental research is required and any successful approach must also prove to be economically viable.

\section{Summary}

This paper deals with autonomous excavation which implies automation of a number of functions related to earthmoving that are currently performed by human operators. It summarizes (1) the necessary functions to be automated in order to benefit from autonomous excavation, (2) the history of the research work that has been carried out towards this purpose, (3) the wide range of research activities that are pertinent to the subject, and (4) a list of references that can be traced for learning the technical content of the related material as well as getting in touch with the people/organizations that are active on the subject.

\section{References}

[1] Bernold, L.E. (1991) Experimental Studies on Mechanics of Lunar Excavation, J. of Aerospace Engineering, 4(1), 9-22.

[2] Blouin, S, Hemami, A and M. Lipsette (2001), "Review of Resistive Force Models for Earthmoving Processes", Journal of Aerospace Engineering, Vol. 14(3), 102-111

[3] Bradley, D.A. Seward, D.W. (1995), "Developing real-time autonomous excavation-the LUCIE story" Proc. the 34th IEEE Conf. Decision and Control, Volume: 3, 3028-3033

Bullock, D. M., Apte, S. M. and Oppenheim, I. J., (1990), "Force and Geometry Constraints in Robot Excavation, Proc. Space 90 Conf. Part 2, Albuquerque, 960-969.

[4] Cannon, H. (1999), "Extended Earthmoving with an Autonomous Excavator", MS Thesis, Carnegie Mellon University, CMU Technical Report \# CMURI- TR-99-10

[5] Cetto, J.A. and Koivo, A.J. (1995), “Controller for Excavator During Digging Operations”, IASTED Int. Conf. Robotics and Manufacturing, Cancun, (Mexico)

[6] Corke, P. I., Winstanley, G. J., Roberts, J. M. (1997) "Dragline modeling and control”,

[7] Proc. IEEE Conference on Robotics and Automation

[8] Ha, Q.P., Santos, M., Nguyen, Q, Rye, D. and Durrant-Whyte, H. (2002), "Robotic Excavation in Construction Automation", IEEE Robotics and Automation magazine, Vol. 9(1), 20-28

[9] Ha, Q. P. and Rye, D. C. (2004), "A Control Architecture for Robotic Excavation in Construction", Computer-Aided Civil and Infrastructure Engineering 19, 28-41

[10] Hemami, A. (1993), "Study of Bucket Trajectory in Automatic Scooping with Load-Haul-Dump Loaders", Trans. Institute of Mining and Metallurgy, 102, sec. A, 37-42.

[11] Hemami, A. (1994a), "Study of Forces in the Scooping Operation of a Mechanical Loader", Transactions of the CSME (Canadian Society of Mechanical Engineers), Vol. 18(3), 191-205.

[12] Hemami, A. (1994b), "Modelling, Analysis and Preliminary Studies for Automatic Scooping", Advanced Robotics, Vol 8(5), 511-529.

[13] Hemami, A. Goulet, S. and Aubertin M. (1994c), "Resistance of Particulate Media to Excavation: Application to Bucket Loading", Int. Journal of Surface Mining and Reclamation, Vol 8, 125-129.

[14] Hurteau, R., St-Amant, M., Laperriere, Y., Chevrette, G.(1992) "Optical Guidance System for Underground Mine Vehicles," Proc. International Conference on Robotics and Automation,

[15] Lever P.A. and Wang F.Y. (1995), "Intelligent excavation control system for lunar mining system”, J. Aerosp. Eng., Vol. 8(1), 16-24

[16] Luengo, O. Singh, S. Cannon, H. (1998), "Modeling and Identification of Soil-Tool Interaction in Automated Excavation”, Proc. IEEE/RSJ International Conference on Intelligent Robots and Systems, Vol. 3, 1900-1906

[17] Mrad, F., Abdul-Malak, M. A., Sadek, S. \& Khudr, Z. (2002), “Automated excavation in construction using robotics trajectory and envelop generation", Engineering, Construction and Architectural Management, Vol. 9(4), 325-335

[18] Marshall, J.A. (2001), “Towards Autonomous Excavation of Fragmented Rock: 
[19] Experiments, Modelling, Identification and Control”, Master's Thesis, Queen's University, Kingston, Ontario, Canada

[20] Mikhirev, P.A. (1986), "Design of Automated Loading Buckets”, Soviet Mining Science, 22(4), PP 292297.

[21] Richardson-Little W. and Damaren C. (2008), "Position Accommodation and Compliance Control for Robotic Excavation", Journal Aerospace Engineering, Vol. 21(1), 27-34

[22] Roberts, J.M., Winstanley, G.J and Corke, P.I. (1999),'Issues for Obstacle Detection for Autonomous Mining and Construction Vehicles", Proc. ISARC 16, Madrid (Spain),

[23] Rowe, P.S. (1999) “Adaptive Motion Planning for Autonomous Mass Excavation”, PhD Thesis, Carnegie Mellon University, CMU Technical Report \# CMU-RI-TR-99-09

[24] Sarata, S., Osumi, H., Hirai, Y and Matsushima, G. (2003), “Trajectory Arrangement of Bucket Motion of Wheel Loader", Proc. ISARC 2003, Eindhoven (Netherlands), 135-140.

[25] Sarata, S, Weeramhaeng, Y and Tsubouchi, T. (2005), "Planning of Scooping Position and Approach Path for Loading Operation by Wheel Loader", Proc. 22nd International Symposium on Automation and Robotics in Construction (ISARC 2005), Ferrara (Italy)

[26] Seward, D, Bradley, D and Bracewell, R. (1988), "The Development of Research Models for Automatic Excavation", Proc. 5th Int. Symp. on Robotics in Construction, Tokyo, Vol. 2, $703-708$.

[27] Seward, D., Bradley, D., Mann, J. and Goodwin, M. (1992), "Controlling an Intelligent Excavator for Automated Digging in Difficult Ground", Proc. 9th Int. Symp. on Aut. and Robotics in Construction (ISARC), Tokyo (Japan) 743-750.

[28] Shi, X, Lever, P.J. A and F-Y Wang, (1996), "Experimental Robotic Excavation with Fuzzy Logic and Neural Networks", Proc. IEEE Int. Conf. Robotics and Automation, Vol.1, 957-962.

[29] Singh, S. (1997), "The State of the Art in Automation of Earthmoving", ASCE Journal of Aerospace Engineering, Vol 10(4), 179-188.

[30] Singh, S. (1991), “An Operation Space Approach to Robotic Excavation”, Proc. IEEE Int. Symp. Intel. Control., NY, 481-486.

[31] Stentz, A., Bares, J., Singh, S., Rowe, R. (1999a), “A Robotic Excavator for Autonomous Truck Loading," Autonomous Robots, Vol. 7(2), 175-186

[32] Stentz. A. Ollis, M., Scheding, S., Herman, H., Fromme, C., Pedersen, J., Hegadorn, T., McCall, B., Bares, J., Moore, R.(1999b), "Position Measurements for Automated Mining Machinery, Report: Robotics Institute (Carnegie Mellon University), and in Proc. of the 1999 International Conference on Field and Service Robotics

[33] Takahashi, H., Kamata, H., Masuyama, T., Sarata, S. (1995), “Autonomous Shoveling of Rocks by Using Image Vision System on LHD, ” Proc. International Symposium on Mine Mechanization and Automation, Golden, Colorado

[34] Takahashi, H., Hasegawa, M. and Nakano, E. (1999), Analysis on the Resistive Forces Acting on the Bucket of a Load-Haul-Dump Machine and a Wheel Loader in the Scooping Task., Advanced Robotics, Vol 13(2), 97-114.

[35] Vähä, P.K., Skibniewski, M.J. and Koivo, A.J. (1991), "Kinematics and Trajectory planning for Robotic Excavation", Proc. Construction Congress 91, Cambridge (MA), 787-793.

[36] Vahed, S.M., Song, S., Dai, J. S., Lam, H. K., Seneviratne L.D. and Althoefer K. (2008), "The Shortest Path of an Excavator Bucket in its Working Zone", Proc.17th IFAC World Congress (IFAC 08), Seoul (Korea), 13822-26

[37] Zweiri, Y.H., Seneviratne, L.D. and Althoefer, K. (2002), "Model-based automation for heavy duty mobile excavator", Proc. IEEE/RSJ International Conf. Intelligent Robots and System, Vol. 3, $2967-$ 2972 